\title{
Germanica
}

\section{La thématique de Don Juan dans Don Juan oder die Liebe zur Geometrie de Max Frisch}

Die Don Juan-Thematik in Don Juan oder die Liebe zur Geometrie von Max Frisch

Thérèse Robin

\section{(2) OpenEdition}

Journals

Édition électronique

URL : http://journals.openedition.org/germanica/2095

DOI : 10.4000/germanica.2095

ISSN : 2107-0784

Éditeur

Université de Lille

Édition imprimée

Date de publication : 1 janvier 1992

Pagination : 111-120

ISSN : 0984-2632

Référence électronique

Thérèse Robin, "La thématique de Don Juan dans Don Juan oder die Liebe zur Geometrie de Max Frisch », Germanica [En ligne], 10 | 1992, mis en ligne le 03 avril 2014, consulté le 06 octobre 2020. URL http://journals.openedition.org/germanica/2095; DOI : https://doi.org/10.4000/germanica.2095

Ce document a été généré automatiquement le 6 octobre 2020.

(c) Tous droits réservés 


\title{
La thématique de Don Juan dans Don Juan oder die Liebe zur Geometrie de Max Frisch
}

Die Don Juan-Thematik in Don Juan oder die Liebe zur Geometrie von Max Frisch

\author{
Thérèse Robin
}

1 Le personnage de Don Juan est bien connu de la littérature de toutes les époques et de tous les pays. Rien que dans les pays de langue allemande, on recense 83 versions de Don Juan, dont 35 pour le $\mathrm{XX}^{\mathrm{e}}$ siècle. Il est donc intéressant de voir que Max Frisch s'est emparé de ce thème récurrent et a commis une pièce de théâtre en 1953, intitulée Don luan oder die Liebe zur Geometrie. Le titre nous fournit déjà une indication sur la conception qu'a Max Frisch de ce personnage. Cette conception n'est pas sans incidences sur la construction même de la pièce et sur le développement de l'action. Traditionnellement, Don Juan est en soi l'incarnation idéale de la séduction qui ne connaît pas de limites ou de limitations. En lui, sont conjugués l'essence de l'homme, l'amour et la mort. Or, dans Die chinesische Mauer de Max Frisch, apparaît déjà le personnage de Don Juan, qui s'élève contre le traitement littéraire traditionnel du thème de Don Juan :

Was immer ich tue oder lasse, alles wird mir verdeutet und verdichtet. (CM, 147)

2 C'est pourquoi nous allons étudier la façon dont l'auteur présente, dans Don Juan oder die Liebe zur Geometrie, le personnage de Don Juan ainsi que les répercussions sur la pièce, sur sa structure et sa problématique. 


\section{I - Le personnage de Don Juan}

\section{1) Un personnage qui évolue}

3 Le personnage de Don Juan chez Max Frisch se démarque profondément du Don Juan traditionnel. En effet, nous avons devant nous un Don Juan dynamique, un Don Juan qui poursuit une évolution personnelle tout au long de la pièce et qui mûrit avec le temps. Dès l'exposition, Don Juan apparaît non pas comme le type du séducteur, de l'homme dominé par ses instincts, mais comme un jeune homme qui fuit devant les femmes. Il est aussi présenté au travers des dialogues des autres personnages, ce qui le rend apte à évoluer. Il n'est pas figé. Son évolution s'accomplit au quatrième acte, où Don Juan est devenu le Don Juan traditionnel, le grand séducteur. Mais l'évolution ne s'arrête pas là, elle se poursuit dans le cinquième acte : Don Juan est marié et père de famille.

Dans toute la pièce donc, Don Juan vieillit ; jeune homme au début (il a 20 ans), c'est un vieillard à la fin de la pièce. Entre le troisième et le quatrième acte, par exemple, treize ans s'écoulent (Don Juan a 33 ans au quatrième acte). L'évolution est double : en même temps qu'il vieillit, qu'il se transforme et passe d'un personnage non classique à un personnage classique, Don Juan s'abandonne à la géométrie.

\section{2) Un personnage qui aime la géométrie}

5 L'amour, caractéristique du personnage de Don Juan, est présent déjà dans le titre. Mais il s'agit de l'amour de la géométrie et non de l'amour pour les femmes. Certes, Don Juan aspire à cet absolu qu'est l'amour, intimement lié à la reconnaissance de soi et de l'autre, de l'identité (il permet à l'un de percevoir la véritable identité de l'autre). Mais cet autre prend une forme peu banale, la géométrie. En effet, Don Juan ne considère pas l'amour pour une femme comme un absolu. Sacralisé par le mariage, ce n'est qu'une illusion en fait, de la malhonnêteté, ce que symbolisent le masque et le déguisement, très importants dans la pièce, au moins au début. L'amour est donc pour Don Juan associé à la sexualité, à l'érotisme. Certains symboles, comme l'étang, l'eau noire, l'obscurité de la nuit, font référence au caractère trouble, anonyme de l'acte sexuel.

6 Au caractère relatif de l'amour pour les femmes, Don Juan oppose le caractère absolu de la géométrie. Celle-ci représente pour lui un idéal, car dépourvue de subjectivité et d'incertitude :

... was heute gilt, das gilt auch morgen, und wenn ich nicht mehr atme, es gilt ohne mich, ohne euch. (DJ., 49)

7 C'est l'exact contraire de l'Eros. Tout problème d'identité y est résolu : toute figure géométrique est identique à elle-même. Après la noce (D.J., 48), Don Juan se consacre encore davantage à la géométrie qu'avant (D.J., 16).

8 Mais se tourner vers la géométrie signifie aussi se couper de la société et vivre en solitaire. C'est pourquoi Don Juan expérimente le temps comme vieillissement non pas de la société, mais de sa propre personne.

\section{3) Don Juan et la société}

9 Don Juan, bien que séparé de la société, volontairement ou non (c'est un personnage marqué par la mort et qui cause la mort de son entourage), est obligé de compter avec 
elle, dans la recherche de sa propre identité. L'un ne va pas sans l'autre. Il entretient des rapports de lutte avec la société, il essaie d'échapper à la dépersonnalisation qu'elle entraîne, mais doit y succomber.

Tout au long de la pièce, Don Juan apparaît comme un solitaire, mais pas à la manière traditionnelle. C'est-à-dire, il est étrange/étranger, dès le début, et au fur et à mesure, est de plus en plus étranger à la société, au monde. L'accumulation de morts autour de lui (son ami, sa fiancée, son père, Don Gonzalo) montre que la vérité est mortelle. Don Juan cherche la vérité et refuse la femme, comme femme et comme représentante de la société. Comme femme, car l'expérience physique de l'amour lui a enseigné la dichotomie naturelle entre homme et femme. Au principe féminin, l'amour, le sexe, il oppose le principe masculin, la géométrie. Il ne veut mener que la vie d'homme, selon le principe masculin, en refusant le principe féminin:

Ich verstehe die Schöpfung nicht. War es nötig, daß es zwei Geschlechter gibt?

(D.J., 66)

11 D'où sa fuite devant la femme, qui l'oblige à devenir, par mépris, le séducteur. Séduire une femme, les femmes, c'est aussi échapper à la femme comme femme. Mais aussi comme représentante de la société, de ses règlements, de ses traditions, de ses us et coutumes (dont la noce est un aspect). Dénoncer le mariage, par exemple, c'est dénoncer le règne de l'illusion dans la société. Le couple Elvira-Don Gonzalo, couple modèle, est un exemple de dénonciation des structures religieuse et sociale (Elvira trompe en fait son mari, bien qu'elle veille à sauvegarder les apparences).

Malgré tout, Don Juan succombe à la femme, à la société. Il vit le monde comme emprisonnement, dont le château est le symbole :

Ich bin ja ihr Gefangener, vergessen Sie das nicht, ich kann ja nicht aus diesem

Schloß heraus [...] (D.J., 86)

Don Juan finit par endosser un rôle qu'il a depuis toujours refusé. La fuite, qui caractérise Don Juan, devant les femmes, devant la société, est devenue fuite psychique, intellectuelle. Don Juan fuit la réalité extérieure pour trouver la réalité intérieure. Il en était même venu à se séparer de son ami, Roderigo, dans sa quête de la vérité :

Einen Freund zu haben, einen Roderigo, der für mich zitterte in dieser Nacht, es

war schön; ich werde fortan für mich selbst zittern müssen. (D.J., 51)

14 Les événements vécus par Don Juan sont en fait le produit de l'image qu'il a de luimême. Il essaie de se réaliser hors de la société, mais l'image qu'il se fait de lui-même entre en conflit avec celle qu'ont les autres de lui. Don Juan finit par devoir n'être plus que l'image qu'ont de lui les autres. La réalité disparaît derrière le rôle qu'il a à jouer. Face à la société, Don Juan est impuissant, de l'impuissance des intellectuels. Toute la problématique de la pièce est là, qui en conditionne la structure.

\section{II - La structure de la pièce}

Le déroulement de l'action amène à se demander quelle est la structure profonde de la pièce de Max Frisch. 


\section{1) Le déroulement de l'action}

16 L'action commence sur un double plan : nous avons D. Elvira et D. Inez d'une part, et Don Juan de l'autre (en arrière-plan). En fait, nous, nous supposons que cet homme en arrière-plan est Don Juan, Max Frisch nous le laisse deviner. Mais l'accent est surtout mis sur le fait que le jeune homme aperçu fuit devant la/les femme(s). Don Juan caché est ensuite le spectateur et l'auditeur invisible des différents dialogues qui suivent et le décrivent comme amateur de géométrie et non de femmes.

L'évolution de Don Juan se fait au fur et à mesure que la pièce se déroule : au quatrième acte, et seulement à ce moment-là, Don Juan est Don Juan, le séducteur, du moins pour les autres. Et au cinquième acte, particularité de la pièce de Max Frisch, Don Juan est devenu un citoyen "normal ", c'est-à-dire, un époux et un père. Il a fini par s'accepter lui-même et va peut-être aussi trouver dans la paternité la réalisation de son être. C'est en tout cas ce que pense Miranda.

\section{2) Essai de structuration}

Comme maints critiques, nous pourrions donc considérer que le temps est l'élément-clé autour duquel toute la pièce est construite, que les trois premiers actes forment un tout, l'action se déroulant pendant une nuit et deux jours, autrement dit, pendant le moment où Don Juan se forge sa "légende ». Mais ensuite, l'image de Don Juan serait coupée en deux, entre le quatrième et le cinquième acte.

19 Ceci nous amènerait, pour une plus grande cohérence, à prendre les quatre premiers actes comme un ensemble au cours duquel Don Juan reçoit une détermination sociale, sa détermination comme Don Juan. Le dernier acte serait alors à comprendre comme essai de vivre une existence normale.

20 Cependant, la solution à un tel déséquilibre serait de concevoir la pièce comme constituée d'une succession de tableaux entre lesquels l'action se déroulerait et serait rapportée dans les dialogues des personnages. Ainsi, l'essentiel serait axé sur la problématique de Don Juan. Ces « rapports » faits par les personnages, comme celui que fait, au cinquième acte, Don Juan de sa vie actuelle, complètent les actes et forment avec eux un tout. D'après leur contenu, les trois premiers actes seraient à définir comme le préambule, le quatrième acte comme le point culminant, et le dernier acte comme l'épilogue.

21 Toutes les difficultés rencontrées lors d'un essai de structuration satisfaisante de la pièce tiennent en réalité à la psychologisation de la pièce. Toute la pièce tourne autour de la problématique existentielle de Don Juan.

\section{3) Don Juan, le pivot de la pièce}

On pourrait faire de Don Juan le seul représentant du «jeu». Tous les autres personnages, quelle que soit leur importance, seraient les participants du «contrejeu ». L'action secondaire peut être vue comme se fondant dans l'action principale pour éclairer d'un autre point de vue, celui de Célestina, le problème de Don Juan, pour le développer d'une autre manière. 

présent ou non. La problématique de la pièce est la problématique existentielle de Don Juan, traitée comme tension entre l'individu et la société. Tous les représentants de la société, c'est-à-dire tous les personnages autres que Don Juan, constituent une psyché collective qui fait de la pièce une pièce de prise de conscience : il s'agit de dévoiler l'inconscient :

Friselis psychologischer Ansatz verwandelt das ursprüngliche Handlungsdrama in ein Bewußtseinsspiel, das gegen den Sinnhorizont des überlieferten Handlungsmodells die Bewußtseinsabläufe seines Protagonisten setzt ${ }^{1}$.

\section{III - L'action interne, part de la problématique de Don Juan}

\section{1) Une action interne}

L'évolution du personnage de Don Juan vers «Don Juan» et sa conception traditionnelle, la distorsion entre rôle et identité conduisent à la psychologisation du protagoniste. L'accent est mis, au contraire de la tradition "Don Juanesque», davantage sur la relation des faits que sur l'action. Le Don Juan de Max Frisch réfléchit sur lui-même, volontairement, et non pas parce qu'il y est obligé, comme chez Molière. En effet, chez Molière, c'est par exemple Sganarelle qui, au troisième acte, invite Dom Juan à parler. Chez Max Frisch, ce personnage n'apparait pas encore au troisième acte, et même l'ami de Don Juan, Roderigo, ne tente pas de lui soutirer des confidences.

C'est pourquoi nous sommes autorisés à parler "d'action interne", autrement dit, d'évolution psychologique. Cette évolution repose sur un processus temporel. Le vécu temporel chez Max Frisch se trouve naturellement déjà dans la problématique traditionnelle de Don Juan. Mais, chez les prédécesseurs de Max Frisch, Don Juan est toujours présenté à une certaine époque de sa vie et reste d'un bout à l'autre de la pièce au même âge. Chez Max Frisch, par contre, le vieillissement de Don Juan est très nettement indiqué : au début, il a vingt ans ; au quatrième acte, c'est un homme de 33 ans, et au dernier acte, il a encore vieilli, d'après une de ses allusions ;

Sind meine Tage nicht kurz genug? (D.J., 84)

Toute la pièce tend au but suivant: Don Juan doit accepter sa paternité; c'est en quelque sorte une capitulation du Don Juan non traditionnel, ressentie comme une perte, mais en même un premier mouvement vers la maturation. Cela signifie que Don Juan s'accepte enfin soi-même, comme il est, accepte enfin la dimension du présent. Il domine sa crainte du vieillissement, dont le processus est rythmé par les saisons: printemps au premier acte, automne au dernier. Le temps extérieur est intériorisé, les deux se correspondent et indiquent un processus, intériorisé, d'identification, de recherche de soi-même. Arrivé au terme de ce processus d'identification, Don Juan atteint une pleine et entière compréhension et reconnaissance de sa personne. Il y parvient en perdant progressivement ses illusions. Le facteur qui déclenche ce processus est la rencontre nocturne au parc avec Anna. A partir de ce moment-là, alors que Don Juan refuse d'épouser Anna, tout se déroule de façon inéluctable. Cette crise de reconnaissance se termine au troisième acte avec une série de morts. La banqueroute et la passion forcenée que lui portent les femmes déclenchent de nouveau un processus de reconnaissance qui prend un tour positif bien que mélancolique. 


\section{2) Un processus d'individualisation} sont : au premier acte, une insatisfaction plus ou moins latente, qui devient un état de distanciation par rapport à soi-même. Aux deuxième et troisième actes, cet état se transforme en crise qui conduit le personnage à mettre en question son individualité et son existence. Le personnage essaie de rompre avec sa vie présente et de changer de manière de vivre. Au quatrième acte, confronté à son environnement, le personnage prend conscience des possibilités d'arriver à se connaître. Au cinquième acte, ce nouveau regard jeté sur la vie et le monde conduit à une acceptation de soi-même, ce qui permet de retrouver définitivement son équilibre psychologique et d'élaborer une existence plus heureuse sur de nouvelles bases, de se réaliser soi-même.

Ce processus d'individualisation se déroule dans la conscience de l'homme, c'est une prise de conscience de l'inconscient. On peut ainsi définir le but recherché par Max Frisch en employant la thématique de Don Juan ${ }^{2}$ :

Dieses Theater zielt auf Entlarvung von individuellen Prozessen und öffentlichen Mechanismen in ihrem Verhältnis zueinander und bezweckt die Bewußtwerdung und die Behebung individueller und öffentlicher Aspekte, die sich als Schwächen oder Mißstände erweisen.

L'intérêt de la pièce ne réside donc pas dans la transformation de Don Juan en Don Juan, mais dans l'évolution intérieure d'un homme, au sens d'un être humain. Il s'agit d'une mise au jour de l'inconscient par le langage. La scène est l'instrument de cette dénudation de l'inconscient: le théâtre réalise ainsi ce que ne peut faire la vie, l'omniprésence de la conscience.

C'est pourquoi ce théâtre est à l'opposé du théâtre traditionnel. Max Frisch ne cherche pas à imiter sur scène la vie. Dans sa pièce de Don Juan il dénonce le théâtre de l'illusion, en faisant par exemple mettre en scène la descente aux Enfers par Don Juan lui-même. Pour cette raison aussi, le lieu de la pièce est le spectateur en personne, et le personnage traditionnel de Don Juan nous importe moins que le Don Juan en nousmêmes. La coupure entre la scène et le monde nous permet de transpercer le monde à travers la représentation scénique, c'est-à-dire d'apprendre à nous connaître. L'espace théâtral de Max Frisch, c'est le "moi ", l'âme. Par le théâtre, Max Frisch s'adresse à l'être humain par l'être humain, pour atteindre le but suivant (CM., 178) :

Auch Euch, mein junger Mann, verbleiben noch immer die Kontinente der eigenen Seele, das Abenteuer der Wahrhaftigkeit. Nie sah ich andere Räume der Hoffnung.

Nous avons par conséquent devant nous un Don Juan à la recherche de sa propre vérité, en quête de rame humaine. La relation entre réalité et mythe, entre identité et rôle est ainsi de toute première importance. Max Frisch nous montre comment le monde se réalise concrètement avec l'individu. 


\section{BIBLIOGRAPHIE}

Stücke 1/2 (Max Frisch), Suhrkamp Taschenbuch 70 et 81, 6e édition, 1980, Francfort: Éditions Suhrkamp (1962).

Don Juan oder die Liebe zur Geometrie (Max Frisch), Édition Suhrkamp 4,13e édition 1978, Francfort: editions Suhrkamp, lère version : 1953.

Gnüg Hiltrud, «Frischs "Don Juan oder die Liebe zur Geometrie"», in Walter Hinck, Die Deutsche Komödie vom Mittelalter bis zur Gegenwart, Dusseldorf, Éditions Bagel, 1977, s. 305-323.

Quenon Jean, Die Filiation der dramatischen Figuren bei Max Frisch, Paris, Société d'édition Les Belles Lettres, 1975, $399 \mathrm{p}$.

\section{ANNEXES}

\section{Abréviations}

C.M. : Die Chinesische Mauer.

D.J. : Don Juan oder die Liebe zur Geometrie.

\section{NOTES}

1. - Gnüg Hiltrud, Frisch. Don Juan oder Die Liebe zur Geometrie, in Hinck Walter, Die Deutsche Komödie vom Mittelalter zur Gegenwart, s. 322.

2. - Quenon, Die Filiation der dramatischen Figuren bei Max Frisch, s. 55.

\section{RÉSUMÉS}

Max Frisch a, en 1953, repris, après maints auteurs, le thème traditionnel de Don Juan, le type même du séducteur, mais en l'agrémentant, d'après le titre, d'un amour, non pour les femmes, mais pour la géométrie. Cette thématique, traditionnelle, mais en même temps non traditionnelle, conditionne toute la pièce, son contenu et sa structure. Difficile à " découper » de manière satisfaisante, la pièce trouve son unité à travers le personnage de Don Juan. Toute la pièce tourne en effet autour de lui, les autres personnages ne prennent de sens que par rapport à lui, l'action secondaire se fond dans l'action principale. Don Juan subit, au fur et à mesure du déroulement de la pièce, une évolution personnelle, il vieillit et mûrit; parallèlement, il s'abandonne à la géométrie au travers de laquelle il essaie de trouver son identité. Ce faisant, il se coupe de la société, à laquelle il tente d'échapper, mais à laquelle il finit par succomber. En refusant les femmes / la femme, il refuse la société, mais aussi le principe féminin. Il ne veut 
qu'être principe masculin. Il doit cependant à la fin admettre son échec et donc cette dichotomie. Cette acceptation finale qui frise la résignation peut malgré tout comporter un aspect positif: c'est en s'acceptant pour ce qu'il est que Don Juan peut se trouver lui-même. Le théâtre de Max Frisch est, même dans une pièce à thème traditionnel, la prise de conscience et le dévoilement de l'inconscient de l'être. Toute réalité extérieure est psychologisée. C'est l'âme humaine qui est «mise en scène ». Don Juan, c'est nous.

1953 nahm Max Frisch nach vielen anderen Schriftstellern das traditionnelle Thema von Don Juan wieder auf, d.h., das Thema des unverbesserlichen Verführers. Jedoch ist dieser Don Juan schon dem Titel nach mit einer Liebe zur Geometrie ausgestattet. Diese gleichzeitig traditionelle und nicht traditionelle Thematik bedingt das ganze Stück, dessen Inhalt und dessen Struktur. Es fällt einem schwer, eine befriedigende Struktur im Stück zu finden. Nur durch die Gestalt des Don Juan erhält die Komödie ihre Einheit. Tatsächlich kreist alles um Don Juan, die anderen Gestalten sind nur in Bezug auf ihn $\mathrm{zu}$ verstehen, die Nebenhandlung mündet in die Haupthandlung ein. Im Laufe des Stückes erfährt Don Juan eine persönliche Entwicklung, er wird älter und reifer; parallel dazu widmet er sich der Geometrie, wodurch er darauf hofft, seine eigene Identität zu finden. Damit trennt er sich von der Gesellschaft, der er zu entfliehen sucht, die aber ihm überlegen ist. Indem Don Juan die Frau/ die Frauen verneint, lehnt er die Gesellschaft ab sowie das Weibliche. Er will nur das Männliche sein. Letzten Endes muß er doch seinen Mißerfolg akzeptieren, die Vereinigung vom Männlichen und vom Weiblichen in sich. Diese Erkenntnis, die auch als Wehmut und Resignation verstanden werden kann, klingt jedoch auch etwas optimistisch; nur indem Don Juan sich akzeptiert, wie er ist, kann er sich selbst finden. Max Frischs Theater (auch in einem Stück mit traditionellem Motiv) stellt die Bewußtwerdung des Ich, des Unbewußten vor. Jede äußere Wirklichkeit wird psychologisiert. Die menschliche Seele wird inszeniert. Don Juan, das sind wir auch.

\section{AUTEUR}

\section{THÉRÈSE ROBIN}

Université Charles-de-Gaulle - Lille III 